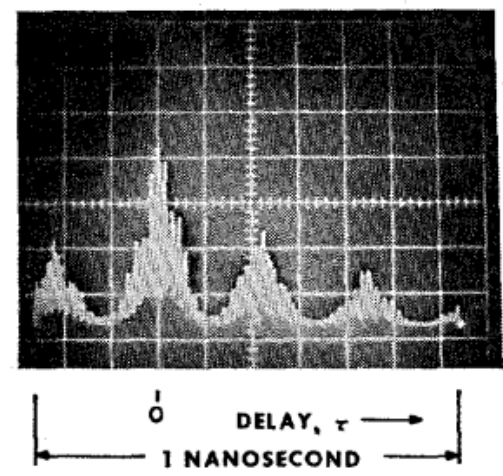

Fig. 4. Correlation peaks in the self-pulsing regime. Total delay is 1 ns indicating a pulse length of $\sim 100$ ps and a repetition rate of $\sim 4 \mathrm{GHz}\left(I / I_{T H} \sim 2.7\right)$.

Paoli and Ripper [1] have suggested that the selfpulsing of GaAs lasers is due to a higher order mode locking in which components of the nonlinear polarization occurring at the second-order difference frequency $\delta^{(2)}(v)$ excite the spiking resonance [9] of the laser. The same laser used in the above experiment was made to selfpulse with nearly 100 -percent modulation at $I / I_{T H} \sim 2.7$ and the resulting correlation pattern is shown in Fig. 4. It should be noted that an intensity interferometer [2] was also used with the diode in this mode of operation to verify that the modulations seen in Fig. 4 do correspond to intensity pulsations. The total delay of Fig. 4 is $1 \mathrm{~ns}$ from which a self-pulsing frequency of $\sim 4 \mathrm{GHz}$ and pulse length of $\sim 100 \mathrm{ps}$ are calculated. Therefore, in this experiment the self-pulsing frequency is 40 to 50 times greater than the second-order difference frequency $\delta^{(2)}(v)$ and it seems unlikely that there is any significant connection between them.

Finally, the value $\delta^{(2)}(v)=84 \mathrm{MHz}$ is small enough that ordinary mode locking in longer $\mathrm{GaAs}$ lasers should be possible and has indeed been achieved by Bogdankevich [10].

\section{ACKNOWLEDGMENT}

The author wishes to acknowledge the helpful suggestions of T. P. Lee and D. Gloge throughout the course of the experiment.

\section{REFERENCES}

[1] T. L. Paoli and J. E. Ripper, "Coupled longitudinal mode pulsing in semiconductor lasers," Phys. Rev. Lett., vol. 22, pp. 1085-1088, May 26, 1969.

[2] D. Gloge and T. P. Lee, "Signal structure of continuously self-pulsing GaAs lasers," IEEE J. Quantum Electron. (Corresp.), vol. QE-7, pp. 43-45, Jan. 1971.

[3] M. Born and E. Wolf, Principles of Optics. New York: Pergamon Press, 1964, p. 500.

[4] H. G. Danielmeyer and H. P. Weber, "Direct measure of the group veloeity of light," Phys. Rev., vol. 3A, May 1971.

[5] T. L. Paoli, J. E. Ripper, and T. H. Zachos, "Resonant modes of GaAs junction lasers-II: High-injection level," IEEE J. Quantum Electron., vol. QE-5, pp. 271-276, June 1969

[6] D. T. F. Marple, "Refractive index of GaAs," J. Appl. Phys., vol. 35, pp. 1241-2142, Apr. 1964.

[7] N. I. Nathan, A. B. Fowler, and G. Burns, "Oscillations in GaAs spontaneous emission in Fabry-Perot cavities," Phys. Rev. Lett., vol. 11, pp. 152-154, Aug. 15, 1963.

[8] A. Yariv, Quantum Electronics. New York: Wiley, 1967, pp. 287-290.

[9 D. E. McCumber, "Intensity fluctuations in the output of CW laser oscillators I," Phys. Rev., vol. 141, pp. 306-322, Jan. 1966.

[10] O. V. Bogdankevich, A. N. Mestvirishvili, A. N. Pechenov, and A. F. Suchkov, "Mode locking in a semiconductor laser with electronic excitation on an internal nonlinear active medium," JETP Lett., vol. 12, pp. 128-129, Aug. 1970.

\title{
The Time Behavior and Spectra of Relaxation Oscillations in a High-Gain Laser
}

\author{
LEE W. CASPERSON AND AMNON YARIV, FELLOW, TEEE
}

\begin{abstract}
It is found that under appropriate conditions the highgain $3.51-\mu$ xenon laser may exhibit relaxation oscillations in the frequency range of 1 to $10 \mathrm{MHz}$. Experimental investigations have been conducted in both the time and frequency domains. Most of the observed features of these fluctuations are in good agreement with theoretical considerations.
\end{abstract}

Manuscript received July 22, 1971. This work was supported by the Air Force Office of Scientific Research.

L. W. Casperson was with the California Institute of Technology, Pasadena, Calif. He is now with the School of Engineering and Applied Science, University of California, Los Angeles, Calif. 90024 .

A. Yariv is with the California Institute of Technology, Pasadena, Calif. 91109.

\section{INTRODUCTION}

$\prod$ HERE are basically two types of transient effects that can occur in laser oscillators. The first of these are the relaxation oscillations. Relaxation oscillations may be considered to include all transients having characteristic times which are long compared to the laser-cavity loop time. The other transients are the ultrashort pulsations, which are fast compared to the cavity loop time. Both types of transients may be obtained in high-gain infrared lasers. Ultrashort pulses have recently been observed in lasers employing the high 
gain $3.51-\mu$ transition in xenon [1], [2]. We report here the observation of spontaneous undamped relaxation oscillations in a $3.51-\mu$ xenon laser. The laser output consists of regular pulsations with repetition rates ranging from about 1-10 MHz. The rate and stability of these pulsations depend on the gain of the laser medium and on the cavity losses.

It has proved to be extremely useful in our study of these transients to make measurements in both the time and frequency domains. In the case of ultrashort pulses the frequency measurements show most effectively the mode pulling and frequency composition of the pulses, while the time-domain measurements indicate the actual structure of the pulse train. These same measurement techniques can also be used to advantage in the study of relaxation oscillations. The time-domain results then are most useful for weakly damped oscillations, but the frequency measurements are essential for studying the strongly damped fluctuations.

\section{SumMary OF THEORY}

The basic features of relaxation oscillations in lasers are well known [3], [4] and relaxation phenomena have been observed in many kinds of lasers. Conventional theoretical treatments begin with a pair of coupled rate equations for the population inversion density and the photon density. The equations may be linearized and solved for the oscillation period $T_{0}$ and the damping time constant $T_{d}$. Such a perturbation solution predicts that the photon density will have a time dependence that may be written in complex form as

$$
q^{\prime}(t) \propto \exp \left[i \frac{2 \pi}{T_{o}} t-\frac{t}{T_{d}}\right]
$$

where $T_{o}$ and $T_{d}$ are given approximately by

$$
\begin{aligned}
& T_{o}=\frac{2 \pi}{\sqrt{\frac{g c_{m}}{\tau}-\frac{1}{\tau t_{c}}}}=2 \pi \sqrt{\frac{\tau t_{c}}{a-1}} \\
& T_{d}=\frac{2 \tau}{g c_{m} t_{c}}=\frac{2 \tau}{a} .
\end{aligned}
$$

Here $g$ is the incremental unsaturated intensity gain constant, $\tau$ is the inversion lifetime, $t_{c}$ is the photon cavity lifetime, and $c_{m}$ is the speed of light in the amplifying medium. The operating point is conveniently indicated by the parameter $a=g c_{m} t_{c}$, which is equal to the ratio of $g$ to its threshold value. At threshold $a=1$.

These rate equation treatments generally assume that the gain per pass is small and that the medium is homogeneously broadened. The validity of these assumptions is not obvious in the case of xenon where the gain may exceed $50 \mathrm{~dB} / \mathrm{m}$ and the Doppler line width is about 30 times as large as the homogeneous line width. A detailed derivation of the period and damping of the relaxation oscillations including inhomogeneous broadening is given in the Appendix. As long as the hole burned in the in-

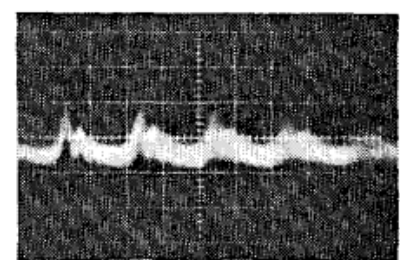

(a)

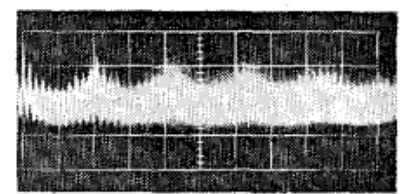

(b)

Fig. 1. Time-domain measurements. (a) Relaxation oscillations at $40 \mathrm{~mA}$ with $0.2 \mu \mathrm{s} /$ div. (b) Relaxation oscillations and ultrashort pulses at $61 \mathrm{~mA}$ with $0.2 \mu \mathrm{s} /$ div.

homogeneous spectrum is not too large, the relaxation behavior is found to be governed to a good approximation by the homogeneous limit given in (1)-(3). Our experiments have been conducted at sufficiently low intensities for the homogeneous results to be expected valid.

Under some circumstances in a xenon laser the damping indicated by (3) may be quite weak. In this case any broadband pump fluctuations or quantum fluctuations [5] may drive the relaxation oscillations at a rate given by (2) with no apparent damping. In practice the modulation may approach 100 percent and the linear approximation breaks down. The damping then manifests itself as a slight frequency instability of the pulsing.

A useful way to study the relaxation oscillations is in terms of the spectrum $\Delta P(\omega)$ of the laser output power fluctuations, which has the approximate form

$$
\Delta P(\omega) \sim \frac{1}{\left(\frac{a-1}{\tau t_{c}}-\omega^{2}\right)^{2}+\omega^{2}\left(\frac{a}{\tau}\right)^{2}} .
$$

This result is obtained from a Fourier transform solution of the rate equations with a broadband driving term. From (4) the spectral width for weak damping is

$$
\Delta \omega \sim \frac{a}{\tau}
$$

thus varying with the gain.

\section{EXPERIMENT}

The laser cavity used in our experiments has two flat mirrors with a spacing of $1.36 \mathrm{~m}$. One of the mirrors could be translated longitudinally to vary the mode frequency. For low values of the gain only one longitudinal mode was above threshold and by adjusting the mirror position the mode could be kept near the gain maximum. The de xenon discharge was $5.5 \mathrm{~mm}$ in diameter and $1.1 \mathrm{~m}$ in length. The pressure was maintained at about $5 \mu$ by means of a liquid nitrogen trap [6]. The cavity losses were varied by introducing attenuators.

An example of a pulsating output with a period of 0.4 $\mu \mathrm{s}$ is shown in Fig. 1(a). Many sweeps are recorded in 
the photograph to give an indication of the degree of stability of the pulsing. Blurring at the left of the picture represents primarily amplifier noise, while the increasing fuzziness to the right of the picture shows that the pulsation period is not strictly constant in this example. Even though the modulation is near 100 percent, the pulse period was found to always be in fairly good agreement with the solution of the linearized equations given by (2). A slight slowing of the pulsing was observed when the modulation was strongest in agreement with the nonlinear equations [3]. The lifetime $\tau$ has been calculated to be $1.35 \mu \mathrm{s}$ [7] and measured values of the gain $g$ and time $t_{o}$ were used. The gain was measured by increasing the known losses until threshold was reached for various values of discharge current. The effective cavity lifetime $t_{c}$ was typically about $1 \mathrm{~ns}$ and could be inferred from the mirror and attenuator losses. By varying $g$ and $t_{c}$, regular pulsations in the frequency range of $1-10 \mathrm{MHz}$ were obtained. The secondary pulses shown in Fig. 1(a) were usually present to some extent but are not well understood. They may be due to coupling to another laser level or to some nonlinearity that has been neglected.

In Fig. 1(b) is shown the simultaneous occurrence of spontaneous relaxation oscillations and ultrashort pulses. The output consists of a train of ultrashort pulses at $26 \mathrm{MHz}$, which is amplitude modulated at $2.2 \mathrm{MHz}$ by the relaxation oscillations. To obtain this result the cavity length was extended to about $6 \mathrm{~m}$.

Some typical data are shown in Fig. 2. These results were obtained from oscilloscope displays of the sort shown in Fig. 1(a). The pulse period is plotted as a function of the number of 50-percent transmission attenuators placed in the cavity with the discharge level held constant. The solid line is a theoretical curve obtained by rewriting (2) as

$$
T_{0}=\frac{2 \pi}{\sqrt{\frac{c_{m}}{\tau}\left(g-g_{n}\right)}}
$$

where $g_{n}$ is the threshold value of the gain for $n$ attenuators, $g=10.6 \mathrm{~m}^{-1}$ is the actual unsaturated gain and the lifetime is taken to be $\tau=1.1 \mu \mathrm{s}$.

These time-domain measurements provide a satisfactory means of studying relaxation oscillations if the damping is very weak. Considerable additional information can be obtained from an examination of the frequency spectrum of the intensity. The frequency-domain measurements are especially useful for strongly damped fluctuations. In this case the spread in frequencies is comparable to the oscillation frequency and the behavior in time is difficult to interpret. The frequency spectrum, however, shows a distinct maximum at the oscillation frequency. The width of this resonance is a measure of the damping.

The apparatus used in the frequency measurements is shown schematically in Fig. 3. The light beam was

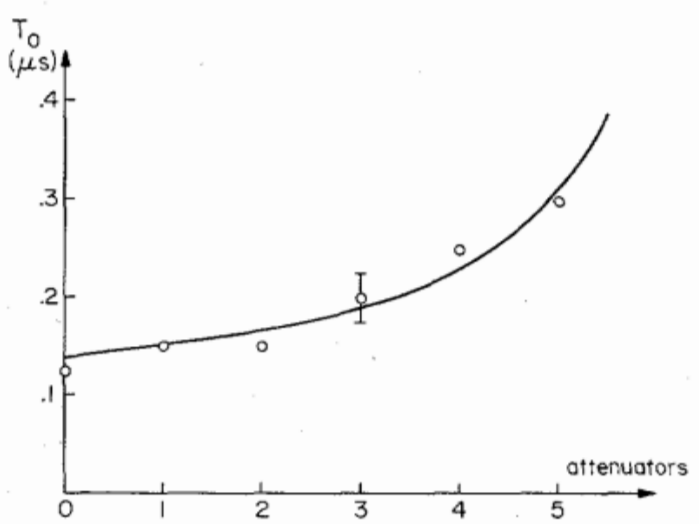

Fig. 2. Pulsation period versus attenuation with a $70-\mathrm{mA}$ discharge. A typical error bracket is shown.

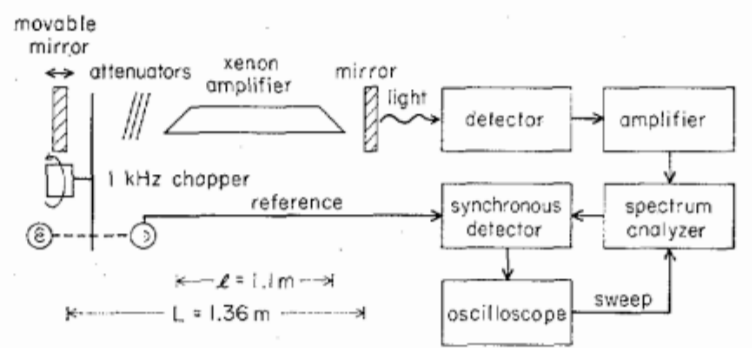

Fig. 3. Experimental setup for frequency-domain measurements.

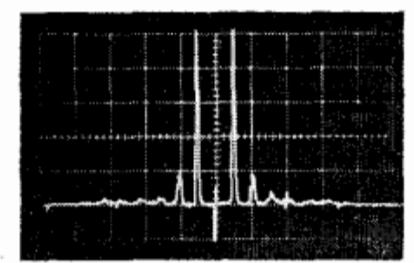

(a)

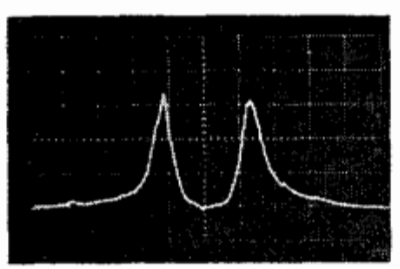

(b)

Fig. 4. Frequency-domain measurements. (a) Beat spectrum of weakly damped pulsations. (b) Strongly damped pulsation with $5 \mathrm{MHz} /$ div dispersion.

chopped and synchronous detection was used. The power spectrum corresponding to the oscillations of Fig. 1(a) is shown in Fig. 4(a). Zero frequency is at the center of the display. The harmonics apparent in the figure are not predicted by (4) and are an indication that because of the strong modulation the linearization has ceased to be valid. At higher values of the gain the damping increases and the harmonics disappear. Such a spectrum is shown in Fig. 4(b). The shift of the peak to higher frequencies as well as the broadening of the spectrum as the gain increases are in agreement with (4). The maximum average power output that could be obtained in the strongly pulsing regime was about $100 \mu \mathrm{W}$ as determined 
with an Eppley thermopile. The peak power was of the order of $1 \mathrm{~mW}$. Pulsewidths as short as $20 \mathrm{~ns}$ could be obtained at the higher currents.

\section{ConCLuSION}

In summary, we have observed relaxation oscillations in a high-gain 3.51- $\mu$ xenon laser. The behavior of the pulsations has been investigated in both the time and frequency domains and was found to be in agreement with a general rate-equation theory. The time domain measurements were most useful in the limit of weak damping and in this regime highly stable pulsations have been observed. The complementary frequency-domain measurements are a more convenient method for obtaining information on the gain-dependence of the damping.

\section{Appendix}

\section{Relaxation Oscillations in Inhomogeneously Broaden ED LASERS}

The purpose of this Appendix is to investigate briefly the effects of inhomogeneous broadening on relaxation phenomena in laser oscillators. Inhomogeneous broadening is common to many lasers, several of which are known to exhibit relaxation oscillations. We obtain here an algebraic equation governing the complex frequency of small-amplitude relaxation oscillations in inhomogeneously broadened lasers.

The starting point for this derivation is the pair of rate equations

$$
\begin{aligned}
\frac{d n(\nu)}{d t} & =P(\nu)-q B\left(\nu, \nu_{l}\right) n(\nu)-\frac{n(\nu)}{\tau} \\
\frac{d q}{d t} & =q \int_{0}^{\infty} B\left(\nu, \nu_{l}\right) n(\nu) d \nu-\frac{q}{t_{c}} .
\end{aligned}
$$

The pumping rate is $P(v), n(v)$ is the population inversion density in the frequency range between $v$ and $v+$ $d v, q$ is the photon density, $v_{l}$ is the laser frequency, $\tau$ is the inversion lifetime, $t_{c}$ is the average photon cavity lifetime, and $B\left(v, v_{l}\right)$ is the frequency dependent Einstein $B$ coefficient. It is assumed in writing the equations in this form that spontaneous emission does not contribute significantly to the photon density, that coupling to other atomic energy levels is unimportant, that spatial variations of the electromagnetic fields-which are substantial in a high gain laser--do not affect the transient behavior of the laser and that coherence effects may be neglected. This treatment is based on one given elsewhere [8].

The behavior of the solutions in the vicinity of the equilibrium point is of greatest interest, so equations (7) and (8) are linearized by the substitutions $q(t)=q_{0}+$ $q^{\prime}(t), n(v, t)=n_{0}(v)+n^{\prime}(v, t)$. The primed quantities are regarded as small perturbations. The first-order results are

$$
\frac{d n^{\prime}}{d t}=-\left(B q_{0}+\frac{1}{\tau}\right) n^{\prime}-B n_{0} q^{\prime}+\left(P-B n_{0} q_{0}-\frac{n_{0}}{\tau}\right)
$$

$$
\begin{aligned}
& \frac{d q^{\prime}}{d t}=q_{0} \int B n^{\prime} d \nu+\left(\int B n_{0} d \nu-\frac{1}{t_{c}}\right) q^{\prime} \\
& +\left(q_{0} \int B n_{0} d \nu-\frac{q_{0}}{t_{c}}\right) .
\end{aligned}
$$

This pair of linear first-order equations is expected to have two independent solutions. The equilibrium point is described by the steady-state solutions

$$
\begin{aligned}
P & =B n_{0} q_{0}+\frac{n_{0}}{\tau} \\
\frac{1}{t_{c}} & =\int B n_{0} d \nu .
\end{aligned}
$$

Equations (11) and (12) may be combined to

$$
\frac{1}{t_{c}}=\int \frac{P d \nu}{q_{0}+\frac{1}{B \tau}} .
$$

If the pumping spectrum is a Gaussian given by

$$
P(\nu)=P_{0} \exp \left[-\left(\frac{2\left(\nu-\nu_{0}\right)}{\Delta \nu_{D}}\right)^{2} \ln 2\right]
$$

where $\Delta_{V_{D}}$ is the Doppler line width and the homogeneous line shape is a Lorentzian given by

$$
B\left(\nu, \nu_{l}\right)=B_{0} \frac{\frac{2}{\pi \Delta \nu_{h}}}{1+\left[\frac{2\left(\nu-\nu_{l}\right)}{\Delta \nu_{h}}\right]^{2}}
$$

where $\Delta v_{h}$ is the homogeneous line width, then (13) may be rewritten as

$$
\frac{1}{t_{c}}=P_{0} \int_{0}^{\infty} \frac{\exp \left[-\left(\frac{2\left(\nu-\nu_{0}\right)}{\Delta \nu_{D}}\right)^{2} \ln 2\right] d \nu}{q_{\circ}+\left(1+\left[\frac{2\left(\nu-\nu_{l}\right)}{\Delta \nu_{h}}\right]^{2}\right) / \frac{2 B_{0} \tau}{\Delta \nu_{h} \pi}} .
$$

If the saturated homogeneous line width is much less than the Doppler line width,

$$
\left(1+\frac{2 q_{0} B_{0} \tau}{\pi \Delta \nu_{h}}\right)^{1 / 2} \Delta \nu_{h} \ll \Delta \nu_{D}
$$

then the pumping spectrum may be replaced by its value at the laser frequency,

$$
P_{l}=P_{0} \exp \left[-\left(\frac{2\left(\nu_{l}-\nu_{0}\right)}{\Delta \nu_{D}}\right)^{2} \ln 2\right]
$$

and (16) simplifies to

$$
\begin{aligned}
\frac{1}{t_{c}} & =\frac{P_{l} B_{0} \tau}{\pi} \int_{-\infty}^{\infty} \frac{d z}{1+\frac{2 q_{0} B_{0} \tau}{\pi \Delta \nu_{h}}+z^{2}} \\
& =\frac{P_{l} B_{0} \tau}{\left(1+\frac{2 q_{0} B_{0} \tau}{\pi \Delta \nu_{h}}\right)^{1 / 2}}
\end{aligned}
$$

where $z=2\left(\nu-{ }_{\nu l}\right) / \Delta y_{h}$ is a normalized frequency. This result determines the steady state value of the photon density $q_{0}$. 
Using (11) and (12), (9) and (10) may be written as

$$
\begin{aligned}
& \frac{d n^{\prime}}{d t}=-\left(B q_{0}+\frac{1}{\tau}\right) n^{\prime}-\frac{P B q^{\prime}}{B q_{0}+\frac{1}{\tau}} \\
& \frac{d q^{\prime}}{d t}=q_{0} \int B n^{\prime} d \nu .
\end{aligned}
$$

These equations may be combined into the second-order differential equation

$$
\frac{d^{2} n^{\prime}}{d t^{2}}+\left(B q_{0}+\frac{1}{\tau}\right) \frac{d n^{\prime}}{d t}=-\frac{P B q_{0}}{B q_{0}+\frac{1}{\tau}} \int B n^{\prime} \cdot d \nu .
$$

Substituting an exponential solution of the form $n^{\prime}(v)=$ $n^{\prime \prime}(v) e^{s t}$ and solving for $n^{\prime \prime}(v)$ leads to

$$
-n^{\prime \prime}=\frac{P B q_{0} \int B n^{\prime \prime} d \nu}{s\left(B q_{0}+\frac{1}{\tau}\right)\left(s+B q_{0}+\frac{1}{\tau}\right)} .
$$

Multiplying by $B(v)$, integrating over frequency, and canceling the factor $\int B n^{\prime \prime} d_{\nu}$ gives

$$
-1=\int_{0}^{\infty} \frac{P d \nu}{q_{0} s\left[1+\frac{1}{B q_{0}}\left(\frac{1}{\tau}\right)\right]\left[1+\frac{1}{B q_{0}}\left(s+\frac{1}{\tau}\right)\right]} .
$$

If the line broadening is strongly inhomogeneous, $P(v)$ may be replaced by $P_{l}$. Then using (15), (22) becomes

$$
\begin{aligned}
&-1=\frac{\Delta \nu_{h} P_{t}}{2 q_{0} s} \int_{-\infty}^{\infty} d z\left\{\left[1+\frac{1+z^{2}}{\left(2 B_{0} q_{0} \tau / \pi \Delta \nu_{h}\right)}\right]\right. \\
&\left.\cdot\left[1+\frac{1+z^{2}}{\left(2 B_{0} q_{0} \tau / \pi \Delta \nu_{h}\right)[1 /(s \tau+1)]}\right]\right\}^{-1} .
\end{aligned}
$$

The integrand in this equation can be expanded in partial fractions and the integrations become elementary. Using (17) for $q_{0}$ the results may be put in the form of the quartic equation

$s^{4} t_{c}{ }^{2} \tau^{2}+s^{3} t_{c}^{2} \tau a^{2}-2 s^{2} t_{c} \tau-2 s t_{c} a^{2}-\left(a^{2}-1\right)=0$

where $a=P_{l} B_{0} \tau t_{c}$ is a parameter, which at threshold is equal to unity. Two of the roots of this equation are nonphysical. It is useful to reduce the number of parameters appearing in the frequency equation by introducing the dimensionless frequency $x=s \tau$ and the ratio $c=$ $\tau / t_{c}$. Then (24) becomes

$x^{4}+a^{2} x^{3}-2 c x^{2}-2 a^{2} c x-\left(a^{2}-1\right) c^{2}=0$.

Equation (25) is the principal result of this appendix. It is valid for small amplitude fluctuations as long as the hole burned by the radiation field in the inhomogeneous spectrum is narrow compared to the overall line width. This equation can be greatly simplified in two important limits. For $a^{4} \ll 2 c$ one finds that the physical roots are given by

$$
x^{2}+a x+c(a-1)=0 .
$$

We refer to this as the homogeneous limit, because it

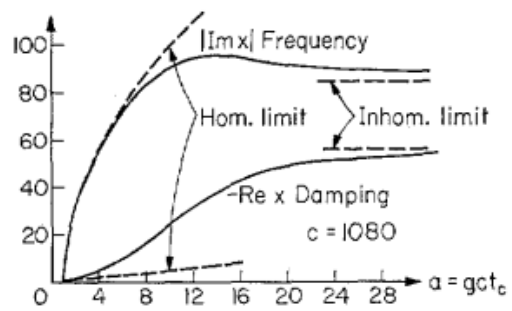

Fig. 5. Real and imaginary parts of the complex frequency $x$ versus $a$ for $c=1080$.

has the same form as one would obtain for a homogeneously broadened medium. With the additional approximation $c(a-1) \gg(a / 2)^{2}$ the solutions of (26) are given in (2) and (3). If the condition $a^{2} \gg c / 2$ is satisfied (for $c \gg 1$ ), then (25) reduces to

$$
x^{3}-2 c x-c^{2}=0 \text {. }
$$

We refer to this as the inhomogeneous limit. Here the complex frequency is independent of the threshold parameter $a$.

In Fig. 5 is a plot of the real and imaginary parts of $x$ found from (25) for $c=1080$, which is a reasonable value for some of our experiments with a xenon laser. The behavior in the homogeneous and inhomogeneous limits is apparent in the figure, where the limiting forms are determined from (26) and (27). The real and imaginary parts of the complex frequency $x$ are proportional to the damping and the frequency of small amplitude relaxation oscillations, respectively. Evidently the oscillation frequency reaches a maximum as the saturation is increased and then approaches the inhomogeneous limit. Eventually, of course, the width of the hole burned in the population inversion spectrum becomes comparable to the Doppler line width as the saturation is continuously increased. When this occurs, the oscillation behavior must return to a homogeneous limit, and the approximation of strongly inhomogeneous broadening made in (23) breaks down. Specifically, this failure is found to occur when the condition $a \ll \Delta_{\nu_{D}} / \Delta_{\nu_{h}}$ ceases to be satisfied. In low-pressure xenon $\Delta_{v_{D}} \simeq 30 \Delta_{\nu_{h}}$.

\section{REFERENCES}

[1] H. H. Kim and H. Marantz, "Continuous self-mode locking of infrared gas lasers," IEEE J. Quantum Electron. (Corresp.), vol. QE-6, pp. 749-750, Nov. 1970.

[2] L. W. Casperson and A. Yariv, "Pulse propagation in a high gain medium," Phys. Rev. Lett., vol. 26, pp. 293-295, Feb. 8, 1971.

[3] R. Dunsmuir, "Theory of relaxation oscillations in optical masers," J. Electron. Contr., vol. 10, pp. 453-458, June 1961.

[4] C. L. Tang, "On maser rate equations and transient oscillations," J. Appl. Phys., vol. 34, pp. 2935-2940, Oct. 1963.

[5] D. E. McCumber, "Intensity fluctuations in the output of CW laser oscillators I," Phys. Rev., vol. 141, pp. 306-322, Jan. 1966.

[6] D. R. Armstrong, "A method for the control of gas pressure in the xenon laser," IEEE J. Quantum Electron. (Corresp.), vol. QE-4, pp. 968-969, Nov. 1968.

[7] P. O. Clark, R. A. Huback, and J. Y. Wada, "Investigation of the de excited xenon laser," Jet Propulsion Lab., Pasadena, Calif., Contract 950803, Final Rep., Apr. 1965.

[8] L. W. Casperson, "Modes and spectra of high gain lasers" Ph.D. dissertation, California Inst. Technol., Pasadena, ch. 6, 1971. 